\title{
Optimal Utilization of IPFC for Congestion Management Using Disparity Line Utilization Factor and Cuckoo Search Algorithm
}

\author{
Akanksha Mishra, G. V. Nagesh Kumar* \\ Department of EEE, GITAM Institute of Technology, GITAM University, Visakhapatnam, India. \\ * Corresponding author. Tel.: 9000573759; email: drgvnk14@gmail.com \\ Manuscript submitted May 21, 2015; accepted December 8, 2015. \\ doi: 10.17706/ijcee.2015.7.6.370-386
}

\begin{abstract}
Recently, due to adoption of power reforms there is a marked increase of contracted power that flows in the transmission line and the spontaneous power exchanges leading to complex power transmission congestion problems. Appearance of FACTS devices specifically IPFC has opened up new opportunities to overcome the congestion problem by increasing the system loadability. Proper placement and tuning of IPFC in this respect is very important for maximum utilization of its benefits. This paper proposes a Disparity Line Utilization Factor (DLUF) for the optimal placement of IPFC to control the congestion in transmission lines. DLUF determines the difference between the percentage MVA utilization of each line connected to the same bus. The IPFC is placed in the lines with maximum DLUF. The multi objective function consisting of the reduction of active power loss, minimization of total voltage deviations, and minimization of security margin with the usage of minimum value of installed IPFC is considered for the optimal tuning of IPFC using Cuckoo Search. The proposed method is implemented for IEEE-30 bus test system under different loading conditions and the results are presented and analyzed to ascertain the effectiveness for the reduction of congestion.
\end{abstract}

Key words: Congestion, cuckoo search algorithm, interline power flow controller, line utilization factor, optimal placement, optimal tuning.

\section{Introduction}

Lately, deregulated electric power industries have changed the way of operation, structure, ownership and management of the utilities. The issue of transmission congestion is more prominent in deregulated and competitive markets, and it needs an appropriate management strategy [1]. In the new competitive electric market, it is now mandatory for the electric utilities to operate such that it makes better utilization of the existing transmission facilities in conjunction with maintaining the security, stability and reliability of the supplied power [2]. Therefore the use of FACTS devices has become inevitable. Economic constraints put a limitation on the number of FACTS devices that can be used. Hence, optimal placement and tuning of FACTS devices in power system is mandatory.

Several authors [1]-[3] have suggested different methods for congestion management. Minguez et al. [4] have propose a method of optimal placement of IPFC such that the load margin is maximized. Mandala et al. [5], proposed a method to determine the optimal location of thyristor controlled series compensators (TCSCs) for Congestion management. The optimal location is determined based on the real power 
performance index and based on the reduction in total system active power and reactive power losses. Reddy et al. [6] has presented the optimal location of FACTS controllers considering branch loading (BL), voltage stability (VS) and loss minimization (LM) as objectives at once using Genetic Algorithm for management of congestion. Acharya et al. [7] propose two new methodologies for the placement of series FACTS devices for congestion management. The overall objective of FACTS device placement can be either to minimize the total congestion rent or to maximize social welfare. Zhang et al. [8] presented an optimal power flow (OPF) control in electric power systems incorporating IPFC with the minimum total capacity of converters of IPFC and minimizing the total active power loss of the system for reducing congestion in the lines. Mohamed et al. [9] has compared three variants of PSO namely basic PSO, Inertia weight approach PSO and constriction factor approach PSO considering a single objective i.e. to minimize the transmission line loss.

FACTS devices are preferred in modern power systems based on the requirement and are found to deliver good solution [10]. Out of all FACTS devices IPFC is considered to be most flexible, powerful and versatile as it employs at least two VSC's with a common DC link. Hence IPFC has the capability of compensating multi transmission line [11]. FACTS devices like TCSC and SSSC are also placed on the most congested line. However, IPFC is a device connected to multiple transmission lines. In its simplest form it has at least two converters placed on two transmission lines connected to a common bus. Proper placement of IPFC is therefore a subject to be analyzed. Location and tuning of FACTS devices in the power system is one of the important issues and hence optimal placement and tuning of IPFC has been proposed based on a previous study [12], [13]. Optimal tuning and placement of IPFC has been done to obtain various objectives [14], [15]. Nature inspired algorithms are among the most powerful algorithms for optimization [16]. Cuckoo Search is inspired from brooding parasitism of cuckoo species in nature. It has only two parameters pa and population size. The convergence rate of the cuckoo search is considered to be insensitive to parameter pa [17]. Thus it need not be fine-tuned for a specific problem Cuckoo Search Algorithm has been found to be superior to PSO and GA with almost exponential convergence rate [18], [19].

In this paper, a Line Utilization Factor (LUF) is used for the determination of congestion of a single transmission line. However, IPFC is the multiline FACTS device. Hence LUF is not a sufficient index for obtaining the location for placement of IPFC. The difference of Line Utilization Factors between two lines has been used for the determination of the optimal location of IPFC. It gives an estimate of the difference of the percentage of line being used for the power flow. Thus all the line pairs of a common bus are ranked in terms of line congestion. The IPFC is placed in the lines with maximum value of DLUF to reduce congestion and power loss in the system. A multi Objective optimization has been formulated for optimal tuning of IPFC using Cuckoo Search algorithm. The multi objective function comprises forthe reduction of active power loss, minimization of total voltage deviations and minimization of security margin with the usage of minimum value of installed IPFC. Tuning of IPFC for the reduction of loss further reduces line congestion. The reduction of Voltage deviation and security margin ensure power quality and system security. The proposed method is implemented and tested on an IEEE 30 bus system with different loading conditions.

\section{IPFC Modelling}

IPFC consists of at least two back to back DC-AC converters connected by a common DC link [20]. $V_{i}, V_{j}, V_{k}$ are complex voltages at bus $i, j, k$ respectively. $V_{l}=V_{l}\left\llcorner\theta_{l}(l=i, j, k)\right.$ and $V_{l}, \theta_{l}$ are the magnitude and angle of $V_{l}$. $V s e_{i n}$ is the complex controllable, series injected voltage source. It shows the series compensation of the series converter. Vse ${ }_{i n}$ is given by $V s e_{i n}=V s e_{i n}\left\llcorner\theta s e_{i n}(n=j, k) . V s e_{i n}\right.$ and $\theta s e_{i n}$ are the magnitude and angle of $V s e_{\text {in }}$.

The basic model of IPFC, as shown in Fig. 1 consisting of three buses $i, j$ and $k$. Two transmission lines are 
connected with the bus $i$ in common. The equivalent circuit of the IPFC with two converters is represented in Fig. 2. $Z s e_{i n}$ is the series transformer impedance. $P s e_{i n}$ is the active power exchange of each converter via the common DC link. $P_{i}$ and $Q_{i}$ has given in equations (1) and (2) are the sum of the active and reactive power flows leaving the bus $i$. The IPFC branch active and reactive power flows leaving bus $n$ are $P_{n i}$ and $Q_{n i}$ and expressions are given in the equation (3) and (4). $I_{j i} I_{k i}$ are the IPFC branch currents of branch $j-i$ and $k-i$ leaving bus $j$ and $k$, respectively [21].

$$
\begin{gathered}
P_{i}=V_{i i i}^{2}-\sum_{n} V_{i} V_{n}\left[g_{i n} \cos \left(\theta_{i}-\theta_{n}\right)+b_{i n} \sin \left(\theta_{i}-\theta_{n}\right)\right]-\sum_{n} V_{i} V s e_{i n}\left[g_{i n} \cos \left(\theta_{i}-\theta s e_{i n}\right)-b_{i n} \sin \left(\theta_{i}-\theta s e_{i n}\right)\right](1) \\
Q_{i}=-V_{i}^{2} b_{i i}-\sum_{n=j, k} V_{i} V_{n}\left[g_{i n} \sin \left(\theta_{i}-\theta_{n}\right)-b_{i n} \cos \left(\theta_{i}-\theta_{n}\right)\right]-\sum_{n=j, k} V_{i} V s e_{i n}\left[g_{i n} \sin \left(\theta_{i}-\theta s e_{i n}\right)-b_{i n} \cos \left(\theta_{i}-\theta s e_{i n}\right)\right](2) \\
P_{n i}=V_{n}^{2} g_{n n}-V_{i} V_{n}\left[g_{i n} \cos \left(\theta_{n}-\theta_{i}\right)+b_{i n} \sin \left(\theta_{n}-\theta_{i}\right)\right]+V_{n} V s e_{i n}\left[g_{i n} \sin \left(\theta_{n}-\theta s e_{i n}\right)-b_{i n} \cos \left(\theta_{n}-\theta s e_{i n}\right)\right](3) \\
Q_{n i}=-V_{n}^{2} b_{n n}-V_{i} V_{n}\left[g_{i n} \sin \left(\theta_{n}-\theta_{i}\right)-b_{i n} \cos \left(\theta_{n}-\theta_{i}\right)\right]+V_{n} V s e_{i n}\left[g_{i n} \sin \left(\theta_{n}-\theta s e_{i n}\right)-b_{i n} \cos \left(\theta_{n}-\theta s e_{i n}\right)\right](4) \\
g_{i n}+j b_{i n}=1 / z s e_{i n}=y s e_{i n}, g_{n n}+j b_{n n}=1 / z s e_{i n}=y s e_{i n}, g_{i i}=\sum_{n=j, k} g_{i n}, b_{i i}=\sum_{n=j, k} b_{i n}
\end{gathered}
$$

Assuming lossless converter, the active power supplied by one converter equals the active power demanded by the other, if there are no underlying storage systems

$$
\operatorname{Re}\left(V s e_{i j} I_{j i}^{*}+V s e_{i k} I_{k i}^{*}\right)=0
$$

where the superscript* denotes the complex conjugate.

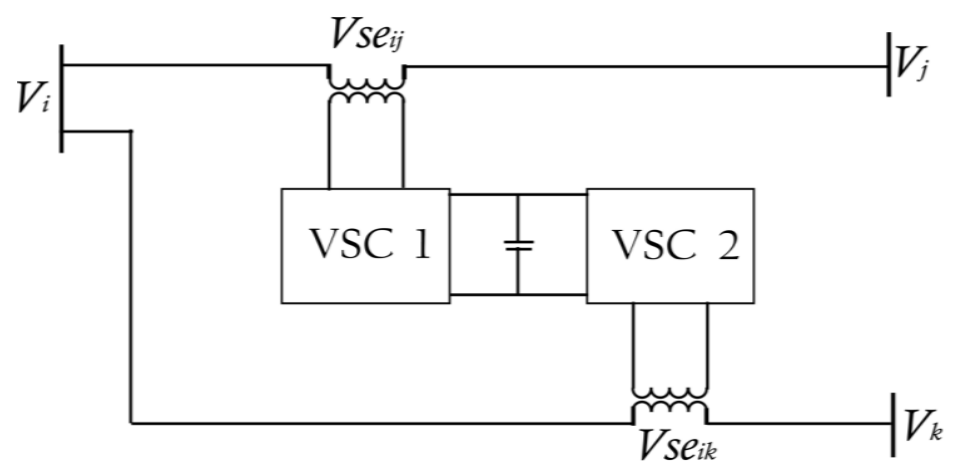

Fig. 1. Basic model of IPFC.

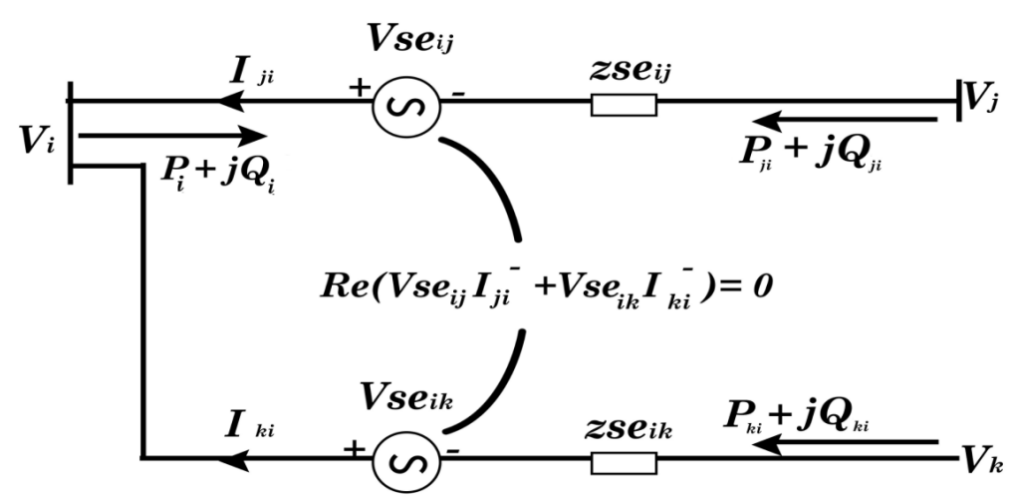

Fig. 2. Equivalent circuit of IPFC. 


\section{Disparity Line Utilization Factor}

Line Utilization Factor is an index used for determining the congestion of the transmission lines. It is given by equation (6)

$$
L U F_{i j}=\frac{M V A_{i j}}{M V A_{i j \max }}
$$

where,

$L U F_{i j}$ : The Line utilization factor $(L U F)$ of the line connected to bus $i$ and bus $j$.

$M V A_{i j(\max )}$ : Maximum MVA rating of the line between bus $i$ and bus $j$.

$M V A_{i j}$ : The Actual MVA rating of the line between bus $i$ and bus $j$.

LUF gives an estimate of the percentage of the line being utilized and is an efficient method to estimate the congestion in a line. For placement of IPFC, there should be at least two lines connected to a common bus. Therefore LUF is not sufficient for Placement of IPFC. Taking into consideration, the fact that IPFC can directly transfer real power via the common DC link, it has the capability to transfer power demand from overloaded to under loaded lines. Hence a new index Disparity Line Utilization Factor is hereby proposed for the optimal placement of an IPFC. DLUF indicates the difference between the utilization of the lines. It gives an estimate of the difference of the percentage of the line being used for the power flow. All the lines are first ranked in descending order of their line utilization factors. The line which has the first rank is considered to be the most congested line. DLUF is calculated for all the lines connected to the line with the highest congestion. All the line pairs connected to the same bus are ranked on the basis of DLUF. The line set that has the highest value of DLUF is considered to be the optimal location of IPFC for Congestion Management. Assuming both lines of same rating:

$$
D L U F_{(i j)-(i k)}=\left|\frac{M V A_{i j}-M V A_{i k}}{M V A_{\max }}\right|
$$

where,

$D L U F_{(i j)-(i k)}$ is the Disparity line utilization factor $(D L U F)$ of the line set $i-j$ and $i-k$ connected to bus $i$ and bus $j$.

$M V A_{i j}$ is the MVA rating of the line between bus $i$ and bus $j$.

$M V A_{\text {MAX }}$ is the maximum $M V A$ rating of line.

$M V A_{i k}$ is the actual $M V A$ rating of the line between bus $i$ and bus $k$.

\section{Step by Step Procedure:}

Step 1: Read line data and bus data.

Step 2: Calculate power flow and LUF of all lines.

Step 3: Calculate DLUF values for all lines in pair with lines ranking highest in congestion.

Step 4: Place IPFC on the lines having the highest value of DLUF.

Step 5: Perform load flow analysis and calculate the LUF of all lines.

\section{Optimal Tuning of IPFC}

An objective function is formulated to find the optimal size of IPFC which minimizes the active power loss, total voltage deviations, and security margin with the usage of minimum value of installed IPFC.

\subsection{Objective Function}

A multi objective function formulated is given in equation (8) 


$$
\min F=\min \sum_{i=1 / \text { to } 4} w_{i} f_{i}
$$

where, $w_{1}, w_{2}, w_{3}, w_{4}$ are weighting factors.

$$
\begin{aligned}
& w_{1}+w_{2}+w_{3}+w_{4}=1 \\
& w_{1}=w_{2}=w_{3}=w_{4}=0.25
\end{aligned}
$$

\subsubsection{Reduction of loss}

The expression for the reduction of active power loss is given in the equation (10) and (11),

$$
\begin{gathered}
\min f_{1}(x)=\sum_{n=j, k}^{l k} P_{\text {loss }} \\
P_{\text {loss }}=\left(\left|V_{i}\right|^{2} G_{i n}-\left|V_{i}\right|\left|V_{n}\right|\left[G_{\text {in }} \cos \theta_{i n}+B_{\text {in }} \sin \theta_{\text {in }}\right]-\left|V_{i}\right|\left|V_{\text {sein }}\right|\left[G_{\text {in }} \cos \theta_{\text {sein }}+B_{\text {in }} \sin \theta_{\text {sein }}\right]\right) \\
+\left(\left|V_{n}\right|^{2} G_{\text {in }}-\left|V_{i}\right|\left|V_{n}\right|\left[G_{i n} \cos \theta_{n i}+B_{\text {in }} \sin \theta_{n i}\right]-\left|V_{n}\right|\left|V_{\text {sein }}\right|\left[G_{\text {in }} \cos \theta_{\text {sein }}+B_{\text {in }} \sin \theta_{\text {sein }}\right]\right)
\end{gathered}
$$

where, $l k$ is the number of transmission lines,

$V_{i}=V_{i} \angle \theta_{i}$ and $V_{n}=V_{n} \angle \theta_{n}$ are the voltages at the end buses $i$ and $n(n=j, k)$.

$V_{\text {sein }}=V_{\text {sein }} \angle \theta_{\text {sein }}(n=j, k)$ is the series injected voltage source of $n^{\text {th }}$ line, se stands for series, $G_{\text {in }}$ and $B_{\text {in }}$ are the transfer conductance and susceptance between bus $i$ and $n(n=j, k)$ respectively. The magnitude and phase angle of the series injected voltage of $V_{\text {seij }}$ and $V_{\text {seik }}$ are determined optimally

\subsubsection{Minimization of voltage deviation}

To have a good voltage performance, the voltage deviation at each bus must be made as small as possible. The Voltage Deviation (VD) can be expressed by the equation (12):

$$
f_{2}(x)=\min (V D)=\min \left(\sum_{k=1}^{N b u s}\left|V_{k}-V_{k}^{r e f}\right|^{2}\right)
$$

$V_{k}$ is the voltage magnitude at bus $k$.

\subsubsection{Minimization of security margin}

The security rate of a system according to the critical state can be expressed as follows in the equation (13).

$$
S M=\frac{\sum_{j \in J_{L}} S_{j} \lim -\sum_{j \in J_{L}} S_{j} \text { initial }}{\sum_{j \in J_{L}} S_{j} \lim }
$$

where, $J_{L}=$ A set contains all load buses.

$S M$ takes a value between zero and one for a system with normal operating condition. A negative value of SM means the system cannot provide the initial load, and the voltage will definitely collapse. Since it is intended to minimize the function, the objective function in the equation (13) is rewritten in the equation (14).

$$
f_{3}(x, u, z)=1-S M=\frac{\sum_{j \in J_{L}} S_{j} \text { initial }}{\sum_{j \in J_{L}} S_{j} \lim }
$$




\subsubsection{Minimization of total capacity of installed IPFC}

The total capacity of the installed IPFC is required for solving the overload on the transmission lines formulated as in the equation (15).

$$
f_{4}(x)=\min \left(P Q_{1}^{2}+P Q_{2}^{2}\right)
$$

where, $P Q$ : the capacity of each VSCs of IPFC.

$$
P Q_{1}^{2}+P Q_{2}^{2}=\left(V s e_{i j}\left(\frac{\overline{V_{i}-V s e_{i j}-V_{j}}}{Z_{i j}}\right)\right)^{2}+\left(V s e_{i k}\left(\frac{\overline{V_{i}-V s e_{i k}-V_{k}}}{Z_{i k}}\right)\right)^{2}
$$

\subsection{Equality Constraints}

$$
\begin{array}{cc}
P_{g i}+P_{i}-P_{D i}=\sum_{j=1}^{n} V_{i} V_{j} Y_{i j} \cos \left(\theta_{i j}+\delta_{j}-\delta_{i}\right) & \forall i \\
Q_{g i}+Q_{i}-Q_{D i}=\sum_{j=1}^{n} V_{i} V_{j} Y_{i j} \sin \left(\theta_{i j}+\delta_{j}-\delta_{i}\right) & \forall i
\end{array}
$$

\subsection{Inequality Constraints}

$$
\begin{gathered}
V_{i}^{\min } \leq V_{i} \leq V_{i}^{\max } \forall i \in \text { loadbus } \\
S_{i n}(V, \delta) \mid \leq S_{i n}{ }^{\text {max }}
\end{gathered}
$$

\subsubsection{IPFC constraints}

$$
\begin{aligned}
& V_{s e}^{\min } \leq V_{s e} \leq V_{s e}^{\max } \\
& \theta_{s e}^{\min } \leq \theta_{s e} \leq \theta_{s e}^{\max }
\end{aligned}
$$

where, $S_{i n}$ is the power injected by the IPFC converters.

\section{Cuckoo Search}

This algorithm is based on the brood parasitic behavior of some cuckoo species along with the L'evy flight behavior of some birds and fruit flies. Some species of cuckoos lay their eggs in common nests while others lay their eggs in the nests of other host birds.

Cuckoo Search considers three rules:

1) Each cuckoo lays one egg at a time, and puts its egg in the randomly chosen nest;

2) The best nests for good quality of eggs will be taken forward to the next generations;

3) The number of available host nests is fixed. The probability of discovery of the cuckoo egg by the host bird is considered as $\mathrm{Pa} \in[0,1]$.

In this case, the host bird can either throw the egg away or abandon the nest, to build a new nest. For simplicity, this last assumption can be approximated by the fraction Pa of the $n$ nests are replaced by new nests (with new random solutions). 
For simplicity, it can be assumed that each egg in a nest represents a solution, and a cuckoo egg represent a new solution. The aim is to use the new and potentially better solutions (cuckoos) to replace a not-so good solution in the nests.

Flow Chart of Cuckoo Search Algorithm:

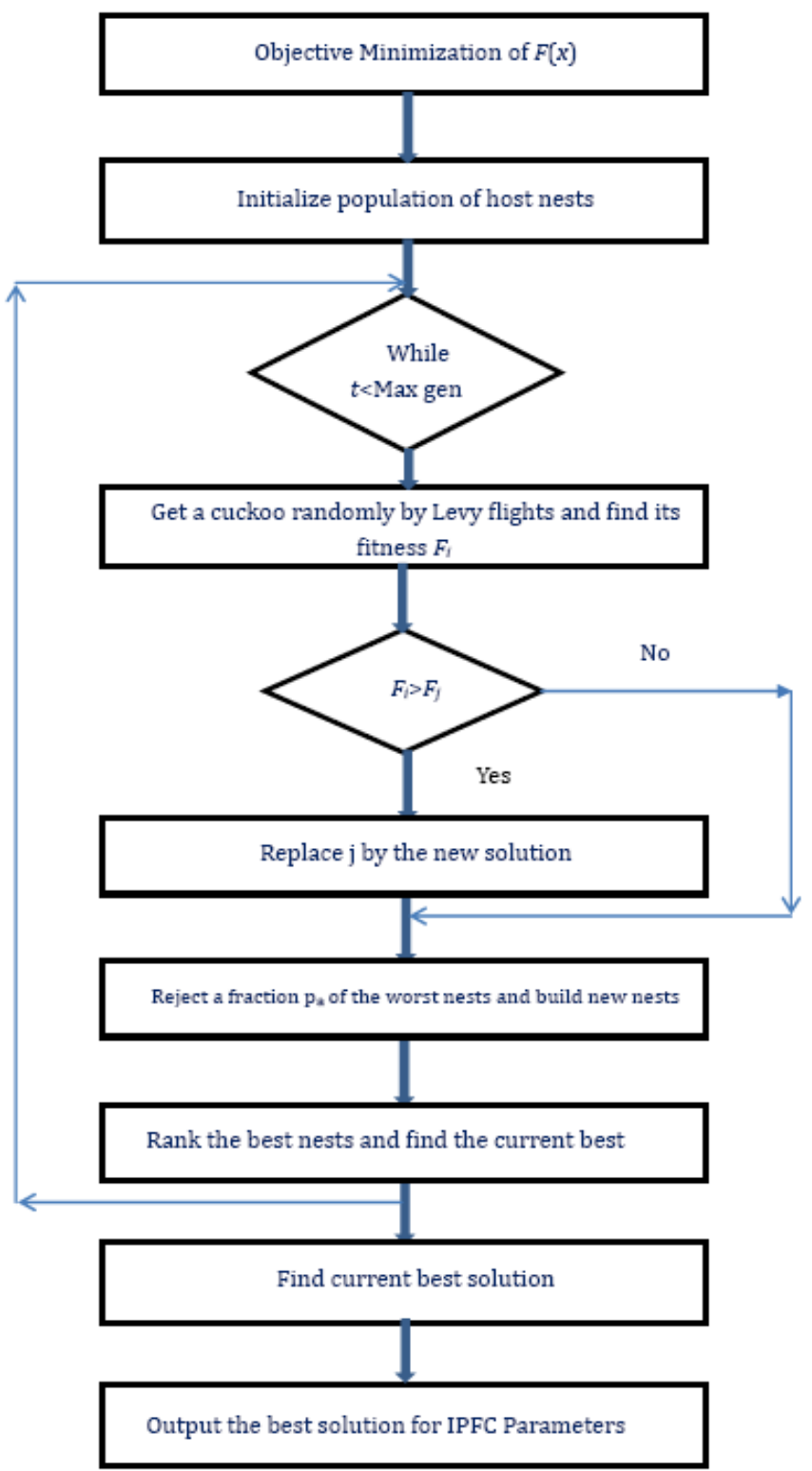

\section{Results and Discussion}

An IEEE 30 bus test system is considered and shown in Fig. 3. In the IEEE 30 bus system bus no. 1 is considered as a slack bus and bus nos. 2, 5, 8, 11, 13 are considered as PV buses while all other buses are load bus. This system has 41 interconnected lines. The IEEE 30 bus test system load flow is obtained using MATLAB Software and the results have been presented. Only load buses are considered for IPFC placement. Equal weights of 0.25 have been considered for all objectives. The results have been analyzed for normal loading, 110\% loading and 125\% loading condition.

LUF values of all the lines without and with Optimal placement of IPFC has been presented in Table 1 and shown in Fig. 4. It is established that line 3-4 is the most congested line connected between the load buses. 
All possible DLUF index calculation for line 3-4 has been shown in Table 2 as test cases. The parameters of Cuckoo Search algorithm used for tuning the IPFC have been mentioned in Table 3. The unturned and the optimally tuned values of IPFC using Cuckoo Search algorithm is given in Table 4. Table 5 compares the real power loss, Voltage deviation, security margin with a smaller capacity of installed IPFC. In Table 6 both active power loss and reactive power loss are compared without IPFC, with untuned IPFC and optimally tuned IPFC. The results for $110 \%$ load and $125 \%$ load have also been presented and analyzed.

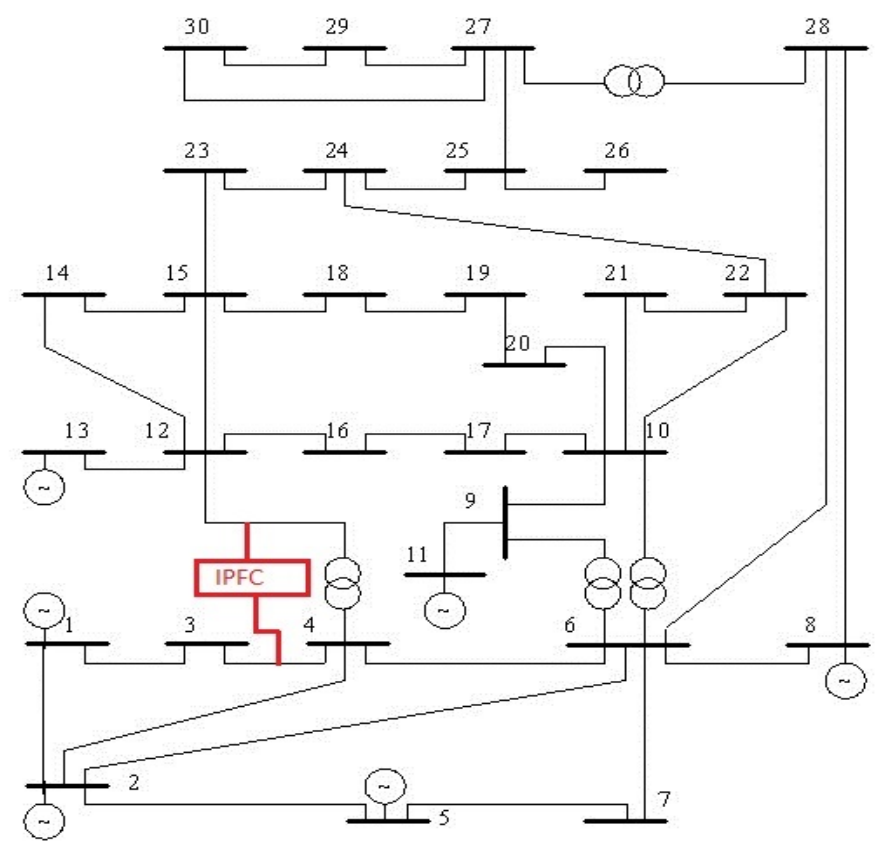

Fig. 3. IEEE 30 bus test system with IPFC installed at the line connected between buses 3-4 and 4-12.

Table 1. LUF Values of All Lines in 30 Bus System

\begin{tabular}{|c|c|c|c|c|c|}
\hline $\begin{array}{c}\text { Line } \\
\text { No. }\end{array}$ & $\begin{array}{c}\text { From Bus } \\
\text { (SB No) }\end{array}$ & $\begin{array}{c}\text { To Bus } \\
\text { (RB No.) }\end{array}$ & $\begin{array}{c}\text { LUF without } \\
\text { IPFC (p.u.) }\end{array}$ & $\begin{array}{c}\text { LUF with un } \\
\text { tuned IPFC (p.u.) }\end{array}$ & $\begin{array}{c}\text { LUF with optimal tuning of } \\
\text { IPFC using cuckoo (p.u.) }\end{array}$ \\
\hline 1 & 1 & 2 & 1.8029 & 1.7979 & 1.7597 \\
\hline 2 & 1 & 3 & 0.9483 & 0.9039 & 0.8870 \\
\hline 3 & 2 & 4 & 0.4939 & 0.4768 & 0.4604 \\
\hline 4 & 3 & 4 & 0.8415 & 0.8334 & 0.8240 \\
\hline 5 & 2 & 5 & 0.8532 & 0.8451 & 0.8362 \\
\hline 6 & 2 & 6 & 0.6473 & 0.6279 & 0.6096 \\
\hline 7 & 4 & 6 & 0.7173 & 0.7439 & 0.6918 \\
\hline 8 & 5 & 7 & 0.2539 & 0.236 & 0.2339 \\
\hline 9 & 6 & 7 & 0.3866 & 0.3835 & 0.3898 \\
\hline 10 & 6 & 8 & 0.397 & 0.4899 & 0.4406 \\
\hline 11 & 6 & 9 & 0.3273 & 0.3042 & 0.3044 \\
\hline 12 & 6 & 10 & 0.2479 & 0.233 & 0.2329 \\
\hline 13 & 9 & 11 & 0.4704 & 0.5146 & 0.5074 \\
\hline 14 & 9 & 10 & 0.6789 & 0.6806 & 0.4988 \\
\hline 15 & 4 & 12 & 0.5284 & 0.5028 & 0.7377 \\
\hline 16 & 12 & 13 & 0.6929 & 0.7521 & 0.1641 \\
\hline 17 & 12 & 14 & 0.1645 & 0.1642 & 0.3861 \\
\hline 18 & 12 & 15 & 0.3858 & 0.3861 & 0.2136 \\
\hline 19 & 12 & 16 & 0.2122 & 0.2133 & 0.0325 \\
\hline 20 & 14 & 15 & 0.0317 & 0.0323 & \\
\hline
\end{tabular}




\begin{tabular}{|l|l|l|c|c|c|}
\hline 21 & 16 & 17 & 0.0868 & 0.0889 & 0.0893 \\
\hline 22 & 15 & 18 & 0.1481 & 0.1485 & 0.1485 \\
\hline 23 & 18 & 19 & 0.037 & 0.0378 & 0.0378 \\
\hline 24 & 19 & 20 & 0.0651 & 0.0644 & 0.0641 \\
\hline 25 & 10 & 20 & 0.1622 & 0.16 & 0.1594 \\
\hline 26 & 10 & 17 & 0.1002 & 0.0978 & 0.0972 \\
\hline 27 & 10 & 21 & 0.2525 & 0.2509 & 0.2500 \\
\hline 28 & 10 & 22 & 0.1296 & 0.1308 & 0.0557 \\
\hline 29 & 21 & 23 & 0.0571 & 0.0564 & 0.0958 \\
\hline 30 & 15 & 23 & 0.0924 & 0.0954 & 0.0553 \\
\hline 31 & 22 & 24 & 0.0545 & 0.0555 & 0.0327 \\
\hline 32 & 23 & 24 & 0.0305 & 0.0328 & 0.0318 \\
\hline 33 & 24 & 25 & 0.0356 & 0.0318 & 0.0432 \\
\hline 34 & 25 & 26 & 0.0434 & 0.0433 & 0.0740 \\
\hline 35 & 25 & 27 & 0.078 & 0.074 & 0.1144 \\
\hline 36 & 28 & 27 & 0.3122 & 0.3013 & 0.0879 \\
\hline 37 & 27 & 29 & 0.1158 & 0.1147 & 0.0424 \\
\hline 38 & 27 & 30 & 0.089 & 0.0882 & 0.0870 \\
\hline 39 & 29 & 30 & 0.0426 & 0.0427 & 0.2023 \\
\hline 40 & 8 & 28 & 0.0799 & 0.0969 & 0.2021 \\
\hline 41 & 6 & 28 & 0.2166 & & \\
\hline
\end{tabular}

Table 2. IPFC Placement on the Basis of DLUF

\begin{tabular}{|c|c|c|c|c|c|}
\hline Case & $\begin{array}{c}\text { Line 1 } \\
\text { SB No- RB No. }\end{array}$ & $\begin{array}{c}\text { Line 2 } \\
\text { SB No- RB No. }\end{array}$ & $\begin{array}{c}\text { LUF Line1 } \\
\text { (p.u.) }\end{array}$ & $\begin{array}{c}\text { LUF Line 2 } \\
\text { (p.u.) }\end{array}$ & DLUF (p.u.) \\
\hline CASE-1 & $3-4$ & $4-6$ & 0.8415 & 0.7173 & 0.1242 \\
\hline CASE2 & $3-4$ & $4-12$ & 0.8415 & 0.5284 & 0.3131 \\
\hline
\end{tabular}

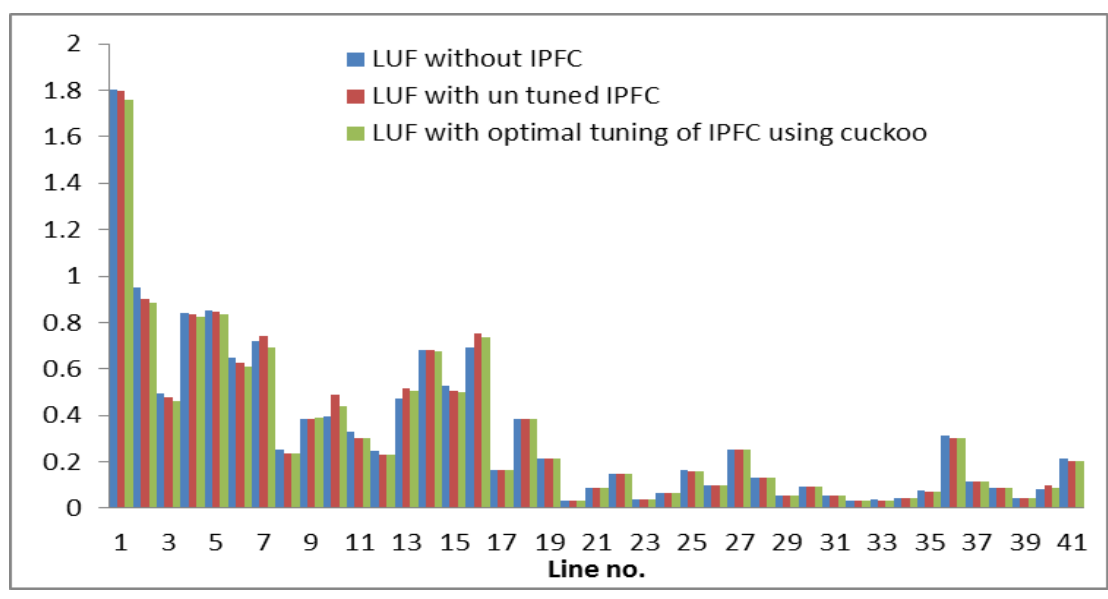

Fig. 4. LUF of lines of 30 bus test system without IPFC and with optimally tuned IPFC.

It is observed from the Table 1, line connected between buses3-4 is the most congested line connected to load bus. In the 30 bus system, two lines have been connected to line 3-4. So, two test cases for IPFC placement have been considered, as shown in Table 2. DLUF has been calculated for each test case, and it is observed that DLUF is the maximum between lines connected to buses 3-4 and 4-12. Hence, lines between buses 3-4 and buses 4-12 have been selected for optimal placement of IPFC. It is observed that placement of IPFC at the location, reduces the congestion in line 3-4 from 0.8415 to 0.8334 . The LUF values before and after placement of IPFC have been compared and shown in Fig. 4. 
After optimal tuning of IPFC using Cuckoo Search algorithm, the congestion in the line reduces to 0.8240 . Fig. 5-Fig. 7 shows the values of the objective functions namely, active power loss, security margin, installed IPFC capacity, and voltage deviation for the different values of Pa. It is observed that for increase in Pa beyond 0.25 the improvement in the value of objective function is very marginal. However, if the rejection of nests on the basis of considering it to be alien is very large, good nests also get rejected in the process. Hence the number of runs required to obtain good results increase if the value of Pa is very large, i.e. the rate of failure is more for a very large value of Pa. With the increase in the number of generations the computation time increases to a very large extent with only marginal improvement in objective function values. Hence, $\mathrm{Pa}=0.25$ and $n=25$ have been chosen for Cuckoo Search Algorithm for optimal tuning of IPFC as listed in Table 4. Fig. 8 shows the Computation Time Required for different values of Pa and Fig. 9 shows the No. of runs for better solution.

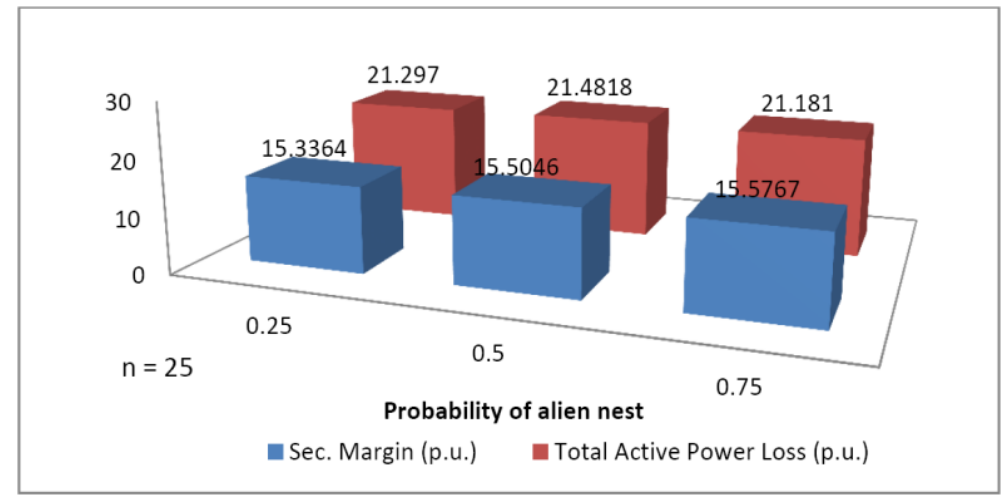

Fig. 5. Security margin and total active power loss vs. probability switch.

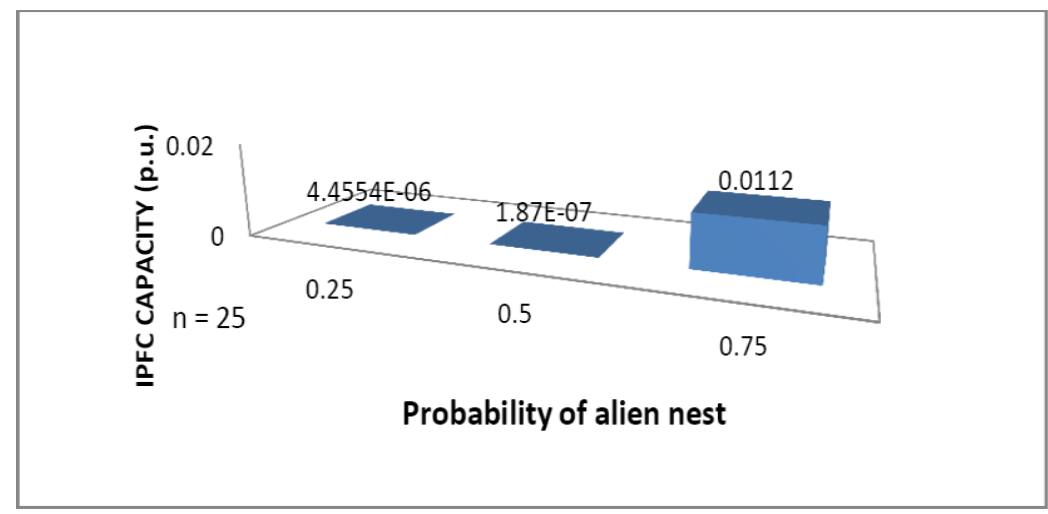

Fig. 6. Installed IPFC capacity vs. probability switch.

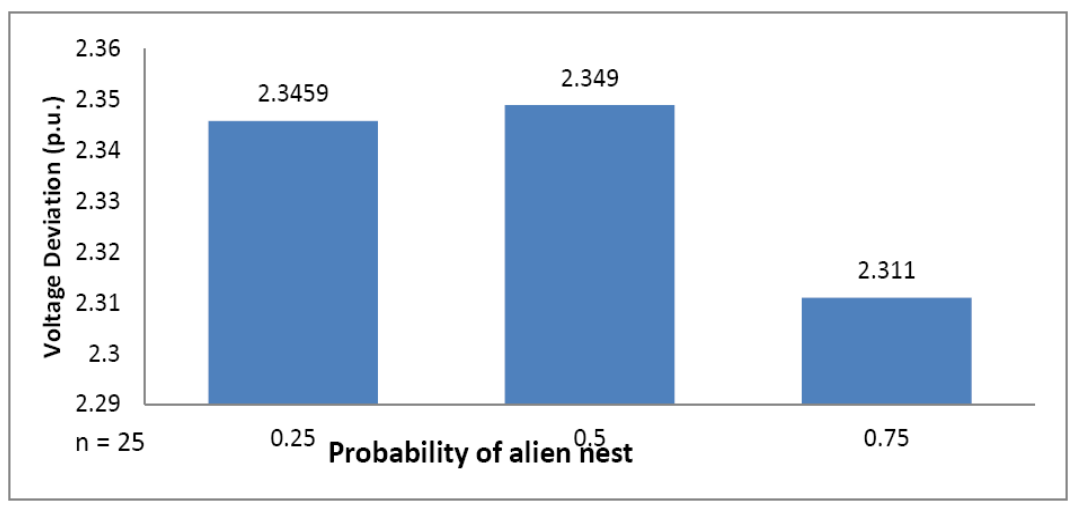

Fig. 7. Voltage deviation vs. probability switch. 


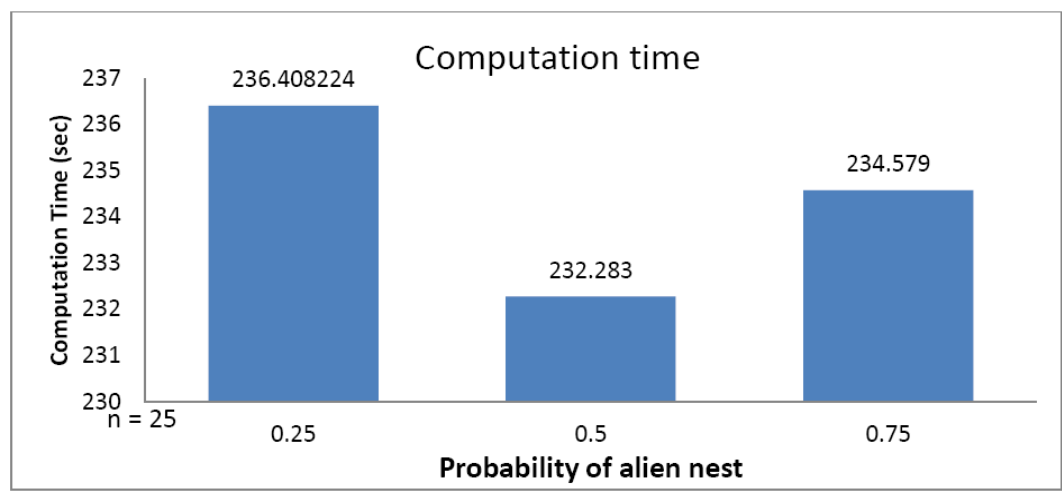

Fig. 8. Computation time required for different values of Pa.

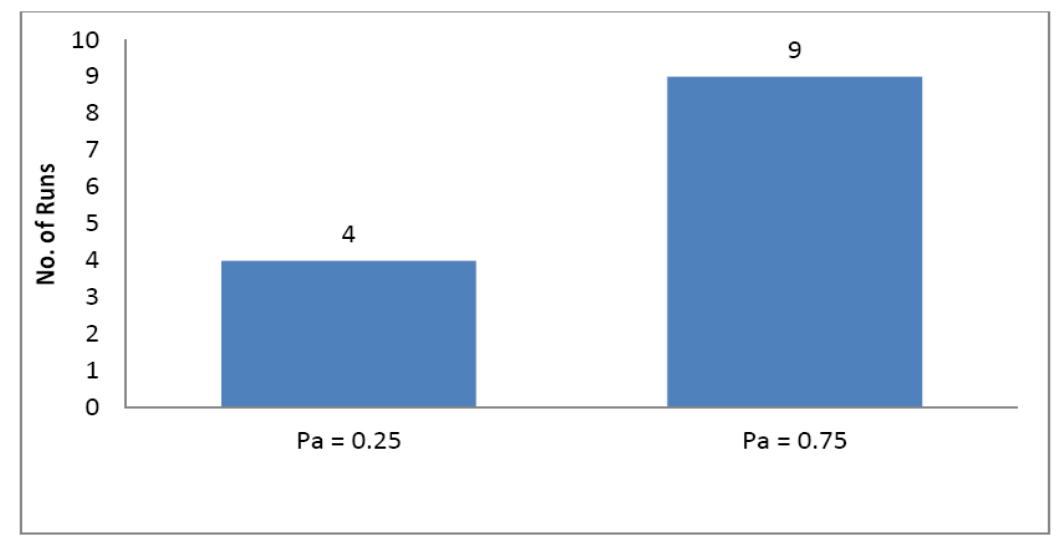

Fig. 9. No. of runs for better solution.

Table 3. Comparative Analysis for Various Population Size

\begin{tabular}{|l|l|l|}
\hline $\mathrm{Pa}=0.75$ & No. of Nests 25 & No. of Nests 50 \\
\hline Active Power Loss (MW) & 21.189 & 20.7797 \\
\hline Security Margin (p.u.) & 15.5767 & 15.7184 \\
\hline Voltage Deviation (p.u.) & 2.311 & 2.1007 \\
\hline Installed IPFC Capacity (p.u.) & 0.0112 & 6.5623 e-8 \\
\hline Computation Time (sec) & $\mathbf{2 3 4 . 5 7 9}$ & $\mathbf{5 5 2 . 8 0 0 9 1 3}$ \\
\hline
\end{tabular}

Table 4. Cuckoo Search Parameters for IPFC Tuning

\begin{tabular}{|l|l|}
\hline Parameter & Value \\
\hline No. of Nests $(n)$ & 25 \\
\hline Probability of finding alien nest $(\mathrm{Pa})$ & 0.25 \\
\hline
\end{tabular}

\subsection{Results for Normal Loading}

Although series devices are primarily supposed to improve power flow in the system, a marked improvement in voltage profile of the buses with optimal placement of tuned IPFC in comparison to voltage profile of the system without IPFC is observed from Fig. 10. Thus, the Voltage Deviation of the overall system is reduced which is also established from Table 6 . Table 6 also shows a reduction in the values of the other objective functions by optimal placement of IPFC. Thus, it is established that by Optimal tuning of IPFC Using Cuckoo Search algorithm the system loss, voltage deviation and also security margin is reduced with the use of minimum capacity of IPFC. The reduction in loss helps in congestion management of the system. The reduction in security margin protects the system against collapse. Lower the capacity of IPFC lower is the cost. Hence, the overall system performance has been improved at a minimum cost. Table 7 
shows the effectiveness of IPFC in the reduction of active and reactive power loss. It also shows the importance of proper placement and tuning of IPFC to be able to completely utilize the IPFC. Fig. 11 shows the fast convergence of Cuckoo Search algorithm in just10 generations.

Table 5. IPFC Parameters before and after Tuning of IPFC

\begin{tabular}{|l|l|l|}
\hline IPFC parameters & Un tuned IPFC & Tuning of IPFC using CS \\
\hline VSe1 (p.u.) & 0.0050 & 0.0012 \\
\hline VSe2 (p.u.) & 0.0100 & 0.0001 \\
\hline Ose1 (degree) & -140.1182 & 180 \\
\hline Ose2 (degree) & 180 & 132.6977 \\
\hline
\end{tabular}

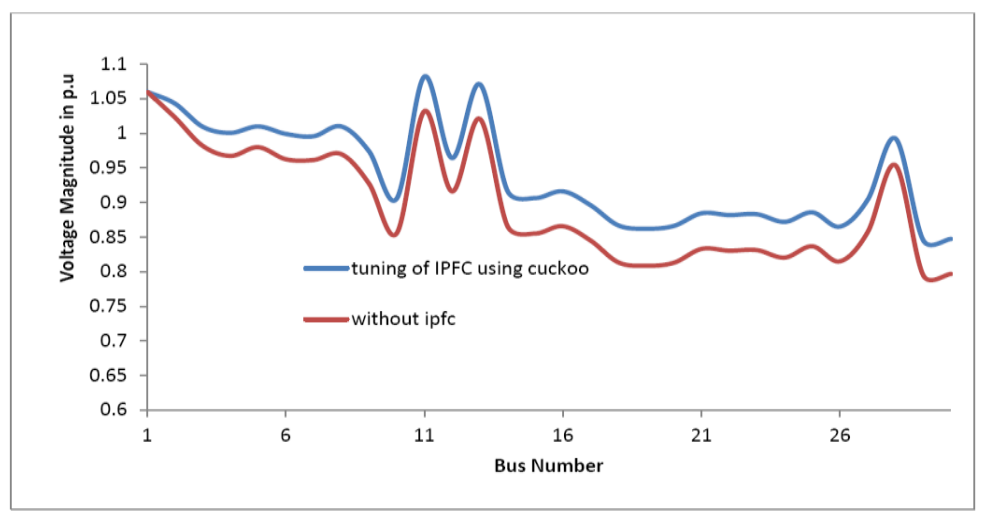

Fig. 10. Voltage profile without and with CS tuned IPFC.

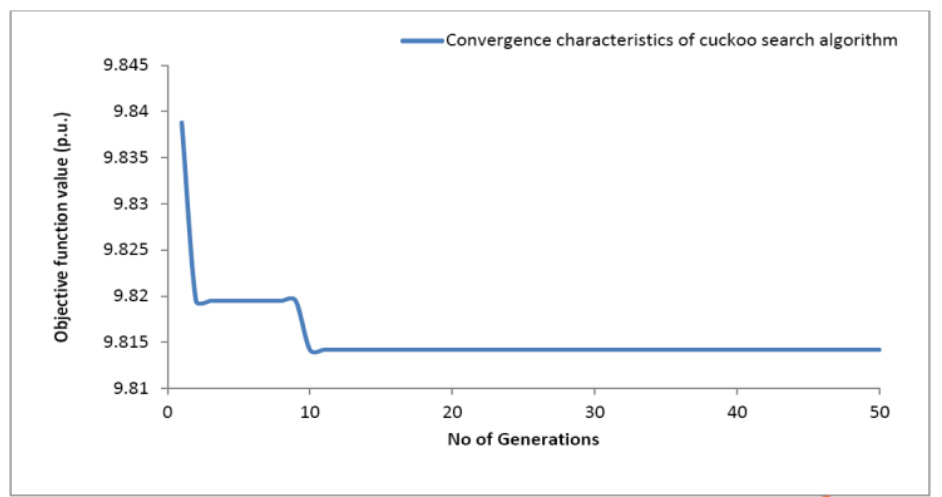

Fig. 11. Convergence characteristics of cuckoo search algorithm with normal loading.

Table 6. Real Power Loss, Voltage Deviation Security Margin and Total Capacity of Installed IPFC with Untuned and with CS Tuned IPFC

\begin{tabular}{|l|l|l|}
\hline Parameters & Untuned IPFC & Tuning of IPFC using CS \\
\hline Real Power losses (MW) & 21.909 & 21.297 \\
\hline Voltage Deviation of all buses (p.u.) & 2.3889 & 2.3459 \\
\hline Security Margin of all lines (p.u.) & 18.2714 & 15.3364 \\
\hline The total capacity of installed IPFC (p.u.) & 0.000406 & 0.0000044554 \\
\hline
\end{tabular}

Table 7. Comparison of Total Real and Reactive Power Loss in the System without IPFC, with Untuned IPFC and with CS Tuned IPFC

\begin{tabular}{|l|l|l|}
\hline & Real Power Losses (MW) & Reactive power losses (MVAR) \\
\hline Without IPFC & 22.941 & 107.370 \\
\hline Un tuned IPFC & 21.909 & 101.334 \\
\hline Tuning of IPFC using CS & 21.297 & 99.616 \\
\hline
\end{tabular}




\subsection{Results for $110 \%$ Loading}

Table 8. IPFC Parameters before and after Tuning of IPFC

\begin{tabular}{|c|l|l|}
\hline IPFC parameters & Un tuned IPFC & Tuning of IPFC using CS \\
\hline VSe1 (p.u.) & 0.0050 & 0.0012 \\
\hline VSe2 (p.u.) & 0.0100 & 0.0169 \\
\hline Ose1 (degree) & -159.8295 & -161.8856 \\
\hline Ose2 (degree) & 180 & -180 \\
\hline
\end{tabular}

Table 9. Comparison of Total Real Power Loss, and Reactive Power Loss without IPFC, with Untuned and with CS Tuned IPFC

\begin{tabular}{|l|l|l|}
\hline & Real Power Losses ( MW) & Reactive power losses ( MVAR) \\
\hline Without IPFC & 27.806 & 127.295 \\
\hline Un tuned IPFC & 26.294 & 118.994 \\
\hline Tuning of IPFC using CS & 25.863 & 117.277 \\
\hline
\end{tabular}

Table 10. Comparison of Real Power Loss, Voltage Deviation Security Margin and Total Capacity of Installed IPFC with Untuned and with CS Tuned IPFC

\begin{tabular}{|l|l|l|}
\hline Parameters & Untuned IPFC & Tuning of IPFC using CS \\
\hline Real Power losses (MW) & 26.294 & 25.8635 \\
\hline Voltage deviation (p.u.) & 2.4609 & 2.4083 \\
\hline Security Margin (p.u.) & 19.4269 & 16.6196 \\
\hline The total capacity of installed IPFC (p.u.) & 0.0002437 & $5.2 \mathrm{e}-5$ \\
\hline
\end{tabular}

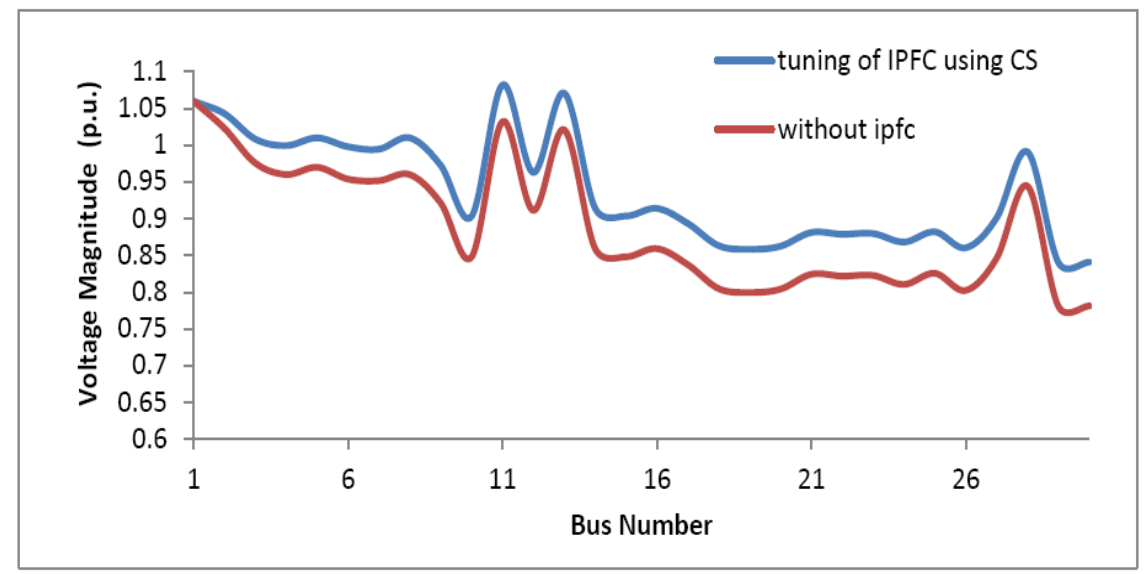

Fig. 12. Voltage profile without IPFC and with CS tuned IPFC under $110 \%$ load condition for IEEE 30 bus test system.

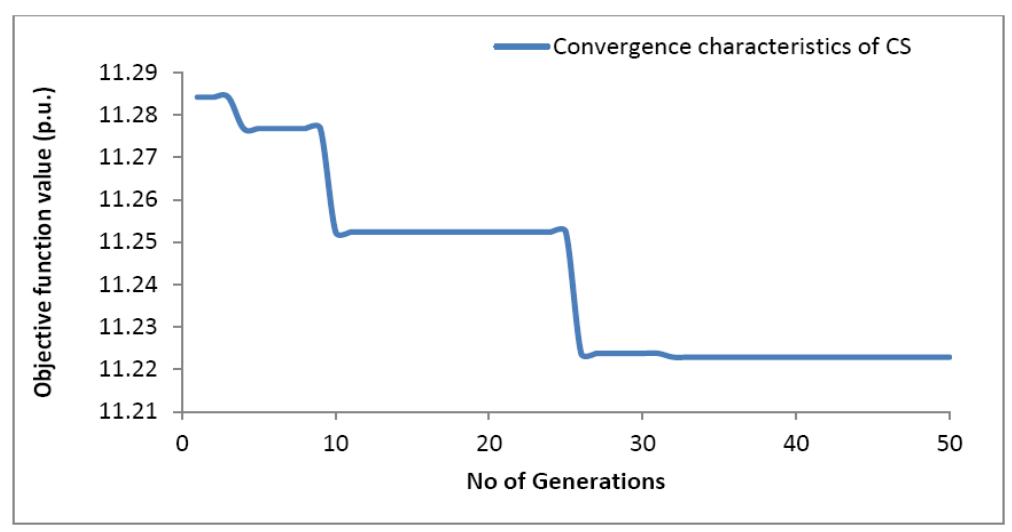

Fig. 13. Convergence characteristics of Cuckoo Search algorithm with $110 \%$ loading. 
Simulation is carried for $110 \%$ load on the IEEE 30 bus test system. It is observed that with the increase in load the total real and reactive power loss increases. Optimal tuning and placement of IPFC has been done to reduce loss, voltage deviation Security Margin and capacity of installed IPFC and the results are presented in Tables 8-10. The voltage profile of the 30 bus system in Fig. 12 with optimally allocated IPFC shows a comparative improvement to that without IPFC. Fig. 13 shows the fast convergence of Cuckoo Search algorithm at 28 generations.

\subsection{Results for $125 \%$ Loading}

Table 11. IPFC Parameters before and after Tuning under 125\% Load

\begin{tabular}{|l|l|l|}
\hline & Untuned IPFC & Tuning of IPFC using CS \\
\hline VSe1 (p.u.) & 0.0050 & 0.0010 \\
\hline VSe2 (p.u.) & 0.0100 & 0.0079 \\
\hline Ose1 (degree) & -167.9689 & 180 \\
\hline Ose2 (degree) & 180 & -174.9885 \\
\hline
\end{tabular}

Table 12. Comparison of Real Power Loss, Voltage Deviation Security Margin and Total Capacity of Installed IPFC with Untuned and with CS Tuned IPFC under 125\% Loading Condition

\begin{tabular}{|l|l|l|}
\hline Parameters & Un tuned IPFC & Tuning of IPFC using CS \\
\hline Real Power losses (MW) & 34.115 & 33.933 \\
\hline Voltage deviation (p.u.) & 2.5861 & 2.5612 \\
\hline Security Margin (p.u.) & 21.2293 & 18.38 \\
\hline The total capacity of installed IPFC (p.u.) & 0.000245 & $9.8008 \mathrm{e}-7$ \\
\hline
\end{tabular}

Table 13. Comparison of Total Real Power Loss, and Reactive Power Loss without IPFC, with Untuned and with CS Tuned IPFC under 125\% Load Condition

\begin{tabular}{|l|l|l|}
\hline & Real Power Losses (MW) & Reactive power losses ( MVAR) \\
\hline Without IPFC & 36.074 & 160.733 \\
\hline Un tuned IPFC & 34.115 & 149.791 \\
\hline Tuning of IPFCusing CS & 33.934 & 149.175 \\
\hline
\end{tabular}

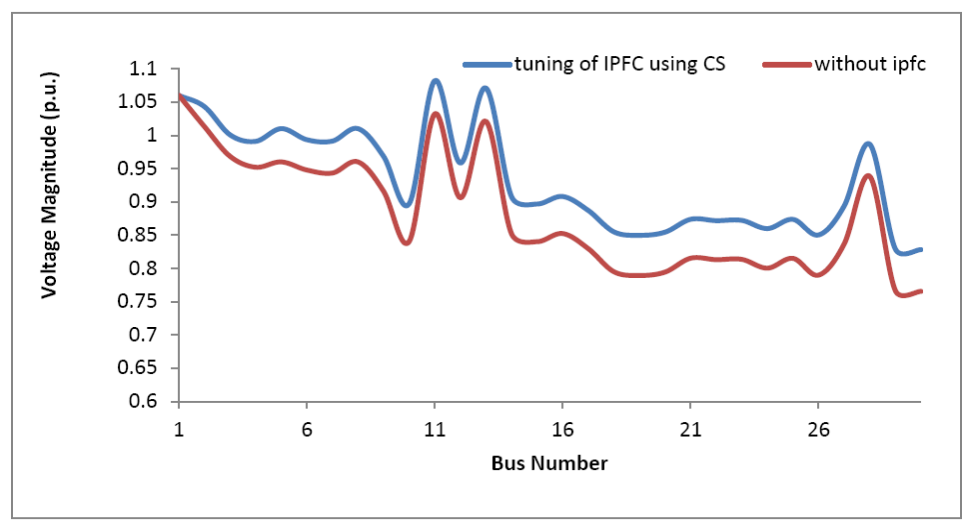

Fig. 14. Voltage profile without IPFC and with CS tuned IPFC under $125 \%$ load condition for the IEEE 30 bus test system.

Simulation Results for 125 \% load have been presented in Tables 11-13. It is observed that the total real and reactive power loss further increases. Optimal tuning and placement of IPFC has been done to reduce loss, voltage deviation Security Margin and capacity of installed IPFC and results are presented in Tables 11-13. Fig. 14 shows improvement in voltage profile without and with IPFC. Convergence characteristics of cuckoo Search Algorithm shows a fast convergence at just 4 generations and shown in Fig. 
15. In Fig. 16, real power loss under different loading conditions has been compared, without IPFC, with untuned IPFC and with optimally tuned IPFC. It is observed that real power loss increases with the increase of loading.

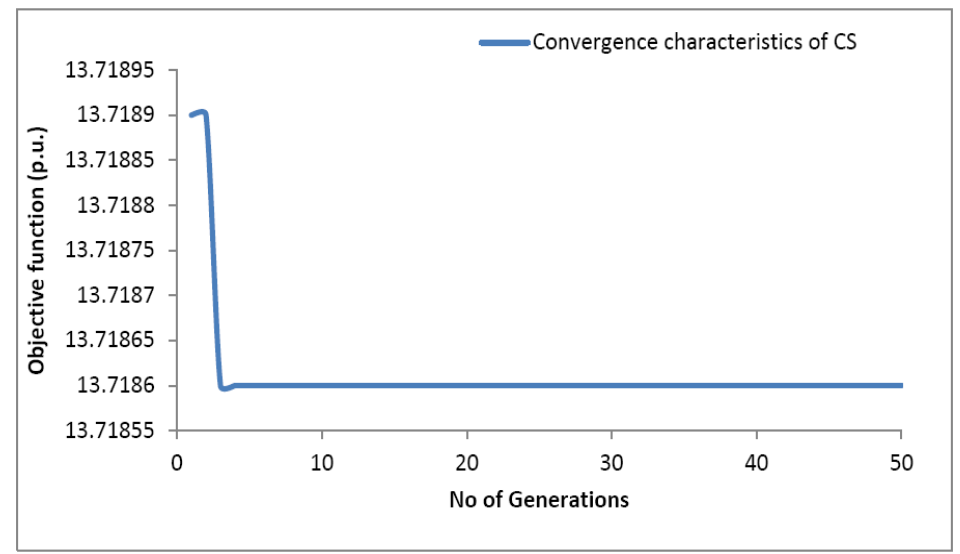

Fig. 15. Convergence characteristics of cuckoo search algorithm with $125 \%$ loading condition.

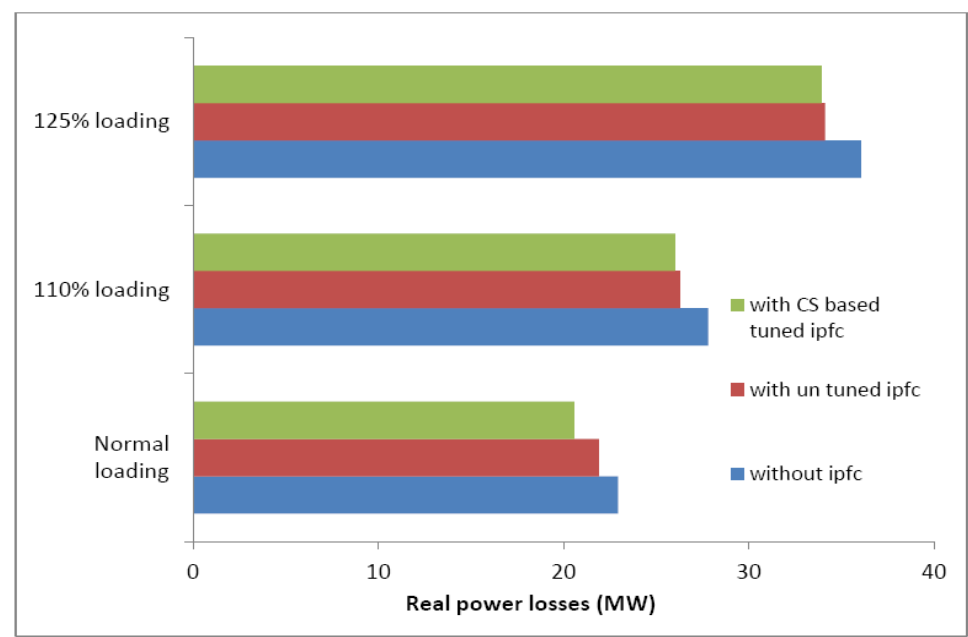

Fig. 16. Real power loss without IPFC with IPFC and with CS based tuned IPFC under normal, $110 \%$ and $125 \%$ loading condition.

\section{Conclusion}

In this paper, a Disparity Line Utilization Factor for the optimal placement of IPFC for congestion management has been implemented. The IPFC is accordingly placed in the lines with highest DLUF value. It has been established that the placement of IPFC using DLUF effectively reduces line congestion and power loss. A multi Objective function comprising of the reduction of active power loss, minimization of total voltage deviations, and minimization of security margin with the usage of minimum value of installed IPFC is considered for the optimal tuning of IPFC using Cuckoo Search algorithm. Effect and importance of the CS parameters for IPFC tuning has been carried. The proposed method is implemented for IEEE-30 bus test system. The results have been presented and analyzed under normal loading, 110\% loading and 125\% loading condition to ascertain the effectiveness of the proposed method on the power system performance. It is observed that placement of IPFC with the proposed methodology causes an effective reduction in congestion in the lines. Simulation results have demonstrated the effectiveness and accuracy of the Cuckoo Search algorithm technique to achieve multiple objectives and to determine the optimal parameters of the 
IPFC under different loading conditions. A reduction in real power loss, voltage deviation, security margin has been achieved with much smaller capacity of installed IPFC. The reduction in loss helps in congestion management of the system. The reduction in security margin protects the system against collapse. Lower the capacity of IPFC lower is the cost. Hence, the overall system performance has been improved at a minimum cost.

\section{References}

[1] Kumar, A., Srivastava, S. C., \& Singh, S. N. (May 2005). Congestion management in competitive power market: A bibliographical survey. Electric Power Systems Research, 76, 153-164.

[2] Singh, S. N., \& David, A. K. (June 2001). Optimal location of FACTS devices for congestion management. Electric Power Syst. Res., 58, 71-79.

[3] Besharat, H., \& Taher, S. A. (June 2008). Congestion management by determining optimal location of TCSC in deregulated power systems. Electrical Power and Energy Systems, 30, 563-568.

[4] Minguez, R., Milano, F., Zarate-Minano, R., \& Conejo, A. J. (Nov. 2007). Optimal network placement of SVC devices. IEEE Trans. on Power Systems, 22(4).

[5] Mandala, M., \& Gupta, C. P. (2010). Congestion management by optimal placement of FACTS device. PEDES and Power India, 1-7.

[6] Reddy, S. S., Kumari, M. S., \& Sydulu, M. (April 2010). Congestion management in deregulated power system by optimal choice and allocation of FACTS controllers using multi-objective genetic algorithm. Transmission and Distribution Conference and Exposition, 1-7.

[7] Acharya, N., \& Mithulananthan, N. (March 2007). Locating series FACTS devices for congestion management in deregulated electricity markets. Electric Power Systems Research, 77(3-4), 352-360.

[8] Zhang, J. (2006). Optimal power flow control for congestion management by interline power flow controller (IPFC). Proceedings of International Conference on Power System Technology (pp. 1-6). Chongqing, China.

[9] Mohamed, K. H., Rama-Rao, K. S., \& Hasan, K. N. M. (2005). Application of particle swarm optimization and its variants to interline power flow controllers and optimal power flow. Proceedings of Transmission and Distribution Conference and Exhibition: Asia and Pacific (pp. 1-7). Dalian, China.

[10] Abdel-Moamen, M. A., \& Padhy, N. P. (2003). Optimal power flow incorporating FACTS devices-bibliography and survey. Proceedings of IEEE PES Transmission and Distribution Conference and Exposition (pp. 669-676).

[11] Hingorani, N. G., \& Gyugyi, L. (2000). Understanding FACTS: Concepts and Technology of Flexible AC Transmission System. IEEE Press.

[12] Teerthana, S., \& Yokoyama, A. (2004). An optimal power flow control method of power system using interline power flow controller (IPFC). Proceedings of the 7th International Power Engineering Conference (pp. 1075-1080).

[13] Kargarian, A., \& Falahati, B. (July 2012). Multiobjective optimal power flow algorithm to enhance multi-microgrids performance incorporating IPFC. Proceedings of Power and Energy Society General Meeting (pp. 1-6). San Diego, CA.

[14] Ram, I. S., \& Amarnath, J. (2013). Optimal setting of IPFC for voltage stability improvement using (GA-GSA) hybrid algorithm. Proceedings of Nirma University International Conference on Engineering (pp. 1-6).

[15] Karthik, B., Alagarasan, I., \& Chandrasekar, S. (2012). Optimal location of interline power flow controller for controlling multi transmission line: A new integrated technique. Frontiers of Electrical and Electronic Engineering, 7(4), 447-458. 
[16] Yang, X. S. Engineering Optimization - An Introduction with Metaheuristic Applications. John Wiley and Sons, Inc.

[17] Yang, X. S., \& Deb, S. (December 2009). Cuckoo search via L'evy flights. Proceedings of World Congress on Nature \& Biologically Inspired Computing (pp. 210-214). India.

[18] Yang, X. S. (2008). Nature-Inspired Metaheuristic Algorithms. Frome: Luniver Press.

[19] Acha, E., Fuerte-Esquivel, C., Ambriz-Perez, H., \& Angeles, C. (2004). FACTS: Modelling and Simulation in Power Networks. John Wiley \& Sons.

[20] Perez, M. A., Messina, A. R., \& Fuerte-Esquivel, C. R. (July 2000). Application of FACTS devices to improve steady state voltage stability. Proceedings of Power Engineering Society Summer Meeting (pp.1115-1120).

[21] Zhang, X. P. (May 2003). Modeling of the interline power flow controller and the generalized unified power flow controller in Newton power flow. IEE Proceedings of Generation, Transmission and Distribution, 150(3), 268-274.

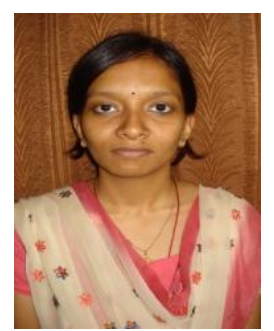

Akanksha Mishra was born in Cuttack, India in 1982. She received her bachelor degree in electrical engineering from the Kalinga Institute of Industrial Technology, Bhubaneswar, India in 2004 and the master's degree in power electronics and drives in 2006 from the same institute. She is presently pursuing her Ph.D. degree at Gandhi Institute of Technology and Management, Visakhapatnam, India. Her research interests are FACTS devices, power electronics and power system stability. She has published several research papers in national and international conferences.

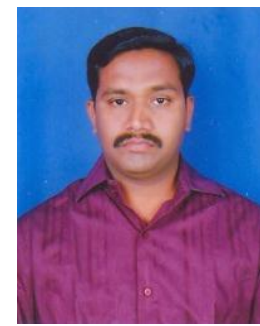

G. V. Nagesh Kumar was born in Visakhapatnam, India in 1977. He received the B.E. degree from the College of Engineering, Gandhi Institute of Technology and Management, Visakhapatnam, India, the M.E. degree from the College of Engineering, Andhra University, Visakhapatnam, India. He received his doctoral degree from Jawaharlal Nehru Technological University, Hyderabad, India. He is also working as an associate professor in the Department of Electrical and Electronics Engineering, GITAM University, Visakhapatnam. His research interests include gas insulated substations, fuzzy logic and FACTS devices. He has published research papers in national and international conferences and journals. 